\title{
Anabases
}

ANABASES Traditions et réceptions de l'Antiquité

4 | 2006

Varia

\section{La magie entre histoire et anthropologie. Relire la contribution d'Henri Hubert au DAGR}

\section{Marcello Carastro}

\section{(2) OpenEdition}

\section{Journals}

Édition électronique

URL : https://journals.openedition.org/anabases/3007

DOI : 10.4000/anabases.3007

ISSN : 2256-9421

\section{Éditeur}

E.R.A.S.M.E.

\section{Édition imprimée}

Date de publication : 1 octobre 2006

Pagination : 251-255

ISSN : 1774-4296

\section{Référence électronique}

Marcello Carastro, «La magie entre histoire et anthropologie. Relire la contribution d'Henri Hubert au

DAGR », Anabases [En ligne], 4 | 2006, mis en ligne le 01 janvier 2012, consulté le 21 septembre 2021.

URL : http://journals.openedition.org/anabases/3007 ; DOI : https://doi.org/10.4000/anabases.3007

(c) Anabases 
Anabases 4 (2006), p. 251-255

\section{La magie entre histoire et anthropologie. Relire la contribution d'Henri Hubert au $D A G R$}

Marcello Carastro

Cosint COMMENT DÉFINIR la magie ? Et en quoi se distingue-t-elle de la religion ? Ce questionnement anime le long article intitulé " Magia " du Dictionnaire des antiquités grecques et romaines. Paru en 1902 1, il a été rédigé par Henri Hubert (1872-1927), un jeune normalien, agrégé d'histoire, qui était titulaire de la chaire de "Religions primitives de l'Europe " à l'École Pratique des Hautes-Études depuis 1901. Les apports de cette contribution et son esprit novateur, qui se fixe pour objectif de définir la magie en associant des données historiques et ethnographiques, doivent être soulignés. Cependant, les acquis de l'histoire et de l'anthropologie invitent aujourd'hui à aborder cette question d'un autre point de vue.

Le choix de confier la rédaction de cet article à Hubert pourrait avoir été inspiré par Salomon Reinach, directeur du Musée des antiquités nationales de Saint-Germain, auprès duquel le jeune historien avait obtenu un poste d'attaché libre, en 1898. Hubert a ensuite poursuivi sa réflexion sur la magie et publié avec Marcel Mauss (1872-1950) un texte de référence sur ce sujet : l'Esquisse d'une théorie générale de la magie, qui est parue dans le septième numéro de l'Année Sociologique, en $1904^{2}$. Leur collaboration, qui transparaît dans le questionnement et la méthode de l'article " Magia », datait de plusieurs années. Hubert et Mauss se sont en effet rencontrés en 1896, au cours de leur formation à l'École Pratique et sont restés très proches ${ }^{3}$. Développant un intérêt

1 Publié d'abord dans le fascicule 31, l'article a ensuite été inséré dans le deuxième volume du tome 3 du Daremberg et SAGLio, 1904, p. 1494-1521.

2 La contribution d'Hubert à l'Esquisse a souvent été négligée, depuis que Cl. Lévi-Strauss l'a insérée au début du recueil d'articles de M. MAUSS, Sociologie et anthropologie, Paris, PUF, 1950. M. Fournier, Marcel Mauss, Paris, Fayard, 1994, p. 104 et suiv. 
commun pour la sociologie des religions, ils ont également cosigné l'Essai sur la nature et la fonction du sacrifice, paru en 1899, ainsi que les Mélanges d'histoire des religions, publiés dix ans plus tard ${ }^{4}$. Avec Mauss, Hubert a rédigé de très nombreux comptesrendus pour la revue l'Année Sociologique et, dès 1897, Émile Durkheim le considérait comme un collaborateur essentiel. Lappartenance à ce cercle intellectuel est manifeste dans la notice "Magia » qui cherche à appréhender la magie et la religion comme des faits sociaux et vise à les distinguer sur le fondement des fonctions qu'elles assurent dans la vie sociale.

La lecture de cet article gagne à être mise en parallèle avec l'Esquisse d'une théorie générale de la magie dont il se rapproche non seulement par l'objectif qu'il se fixe - trouver une définition de la magie qui ne s'applique pas seulement à l'Antiquité grécoromaine, ce qui peut sembler surprenant pour une notice du Daremberg et Saglio mais aussi par la méthode qu'il met en œuvre, faisant référence à des sources ethnographiques. Il est donc possible de penser que les deux auteurs de l'Esquisse avaient commencé à réfléchir ensemble sur ce sujet avant même la rédaction de l'article 5 .

Les deux textes débutent par le constat que, sous l'appellation de magie, sont réunis des phénomènes très divers. Faute de définition scientifique, tous deux se proposent de procéder par tâtonnements et de retenir une " définition provisoire " qui est ensuite mise à l'épreuve, en la comparant à d'autres pratiques qui, tout en étant proches de la magie, s'en distinguent, notamment la religion, la divination, l'alchimie et l'astrologie.

Tout comme l'Esquisse, l'article " Magia » est traversé par la recherche de critères de distinction entre magie et religion. Se fondant notamment sur l'Apologie d'Apulée et la Préparation évangélique d'Eusèbe de Césarée, Hubert propose de caractériser les pratiques magiques par leur but : elles viseraient à modifier l'ordre prévu des événements par des miracles que l'intéressé ne pourrait ou ne voudrait pas demander dans le cadre de pratiques religieuses. L'auteur relève ensuite d'autres traits distinctifs de la magie : son caractère secret, incompréhensible, merveilleux, paradoxal, contre-nature et, enfin, illicite. Car, selon Hubert, la différence entre magie et religion se fonderait sur une distinction d'ordre juridique : la magie serait « essentiellement illicite, sinon criminelle, toujours suspecte et naturellement calomniée » (p. 1496). La prohibition des pratiques magiques instituerait donc une différence essentielle qui les distinguerait des pratiques relevant de la religion. En s'appuyant sur le témoignage de Cicéron, Hubert remarque que l'illégalité de la magie pourrait découler du fait que, dès l'Antiquité, elle était considérée comme un savoir d'origine étrangère. Cette comparaison entre religion

$4 \quad$ Hubert est également l'auteur de deux monographies, Les Celtes et Les Germains, qui seront publiées après sa mort en 1932 et en 1952, la première par les soins de Marcel Mauss et la seconde avec une préface d'Henri Berr.

L'article “Magia " est cité dans l'Esquisse (M. Mauss, op. cit. : 8) parmi les sources utilisées pour l'étude des magies grecques et latines. 
et magie qui traverse l'article aboutit à un dernier critère de distinction : la seconde fait plus souvent appel à des rites sympathiques que la première mais manque de représentations mythiques qui lui soient propres. Son panthéon est un chaos. Elle puise ses éléments dans la religion, mais les désorganise. Elles transforme les figures mythiques en forces. Pour conclure, selon Hubert : " la magie et la religion n'attachent pas la même importance aux mêmes choses. Leur différence vient de celle de leurs fonctions dans la vie sociale» (p. 1521).

Comme le fera également l'Esquisse, l'article " Magia " s'oppose sur plusieurs points aux théories développées par Frazer dans Le rameau d'or, dont une première version avait été publiée en 1890 . Hubert met en évidence l'impossibilité de distinguer les faits magiques des faits religieux sur le fondement de leur objet ou de leur forme, comme Frazer avait cherché à le faire. Il critique également le modèle interprétatif frazérien qui proposait de réserver l'appellation de magie à des rites régis par les lois de sympathie visant à contraindre les puissances divines. Citant l'Essai sur la nature et la fonction du sacrifice, Hubert souligne que " les rites religieux, et le sacrifice en particulier, produisent des effets aussi nécessaires que les rites sympathiques de la magie " (p. 1509). Son argumentation s'appuie sur de nombreuses références tirées de la littérature ethnographique et s'avère plus convaincante que celle présentée dans l'Esquisse. Enfin, Hubert se fonde sur l'exemple de l'alchimie pour montrer que la magie ne se réduit pas, comme le pensait Frazer, à une science fondée sur l'erreur. Au contraire, le fait que les rituels magiques prévoient une "infinité de cérémonies mystiques » montre que la magie est animée par un élan religieux qui la distingue fondamentalement de la science. L'analyse des rituels présentés dans les papyrus magiques conduit ensuite Hubert à mettre en valeur l'attention que la magie accorde au signe efficace, sans se préoccuper de la chose signifiée. Selon sa formule, dans la magie «le mot étouffe le sens » (p. 1519). Cette idée novatrice connaîtra des développements féconds dans l'analyse du discours et de la valeur performative des énoncés 6 .

Les textes antiques auxquels Hubert fait référence datent principalement de l'époque romaine et de l'Antiquité tardive. Il s'agit notamment de l'Apologie d'Apulée, des œuvres de Porphyre, de Plotin et des Pères de l'Église ainsi que des lexiques byzantins. Hubert ne manque pas de mentionner également les sources épigraphiques. Mais, s'il dresse une liste des publications récentes de lamelles en plomb, les devotiones, il ne les utilise pas à l'appui de son argumentation. En revanche, il a amplement recours aux papyrus pour analyser les rites magiques (dans l'Esquisse, ces documents seront d'ailleurs présentés comme des sources essentielles pour accéder aux représentations de la magie grecque). Le choix de privilégier les sources latines découle d'une conviction qu'Hubert énonce dès le début de son article : selon lui, la magie serait devenue un "ensemble cohérent depuis l'entrée des latins dans la civilisation grecque " (p. 1494).

6 Cf. T. TODOROV, “Le discours de la magie”, L’Homme, 13 (1973), p. 38-65 ; S.J. TAMBIAH, Magic, science, religion, and the scope of rationality, Cambridge, Cambridge UP, 1990. 
Cette approche conduit cependant l'auteur à négliger les textes grecs d'époque classique qui sont pourtant essentiels pour saisir comment les Grecs, avant les Romains, ont conçu la magie. Si quelques passages platoniciens et hippocratiques sont cités, l'absence de référence aux Histoires d'Hérodote est particulièrement regrettable dans la mesure où l'historien d'Halicarnasse est le premier à traiter des mágoi, les mages.

Dans un esprit très innovant, Hubert ajoute à ces sources antiques des données ethnographiques en citant des monographies consacrées aux cultures mélanésiennes et australiennes ainsi qu'aux peuples sémitiques, auxquelles il ajoute des données relatives au folklore européen. Ce choix, qui se retrouve dans l'Esquisse, répond à l'objectif que l'auteur s'est fixé : proposer une définition de la magie qui ne se limite pas à l'Antiquité classique. La référence aux travaux ethnographiques lui permet de conforter ses affirmations, à travers une accumulation d'exemples concordants cités dans l'appareillage des notes. Mais le raisonnement anthropologique marque aussi profondément la pensée d'Hubert. D'une part, il le conduit à priver la Grèce ancienne du statut d'exception qui lui était traditionnellement accordé par les historiens, en montrant qu'elle partage de nombreux points communs avec d'autres cultures. D'autre part, les méthodes de l'anthropologie naissante structurent le raisonnement d'Hubert qui organise son exposé, sur un modèle qui sera repris dans l'Esquisse, en centrant successivement son attention sur les acteurs, les représentations, puis les actes appartenant au domaine de la magie. Si les données ethnographiques ont inspiré Hubert dans la rédaction de cet article, on remarquera cependant qu'il ne fait pas référence à la notion mélanésienne de mana qui constituera la clé de voûte de l'Esquisse mais aussi la principale cible de ses détracteurs ${ }^{7}$.

Si l'article d'Hubert n'encourt pas de tels risques, il gagne cependant à être mis en perspective avec les nombreux travaux qui ont été consacrés à la magie depuis un siècle, tant en histoire ancienne qu'en anthropologie. À l'issue des débats qui ont animé ces disciplines, il apparaît aujourd'hui nécessaire de chercher à retracer l'histoire culturelle de cette notion. En effet, dès la fin des années 1950, des anthropologues ont dénoncé le lourd héritage historique qui grève le concept de magie ainsi que son caractère européo-centrique et ont souligné combien il était difficile de l'épurer des représentations qu'il véhiculait ${ }^{8}$. Puisqu'il est apparu que l'histoire de ce concept affecte sa valeur analytique, il n'est plus possible de souscrire à la proposition d'Hubert et de Mauss, qui

7 Dans les premières années de son enseignement "Religions des peuples non civilisés ” à l'EPHE, de 1901 à 1905, Mauss avait consacré une attention particulière à l'étude critique des documents concernant les pratiques magiques en Mélanésie ainsi qu'aux notions fondamentales de la magie. En témoignent les compte-rendus qu'il rédige pour l'Année Sociologique (cf. V. KARADY, Marcel Mauss. Euvres II, Paris, éditions de Minuit, 1974, p. 383-391).

8

Cf. O. Petterson, "Magic-Religion : Some marginal notes to an old Problem ", Ethnos 3-4 (1957), p. 109-119 ; M. et R. WAX, "The notion of Magic", Current Anthropology, 4, 5 (1963), p. 495-518 ; D. Hammond " Magic. A problem in Semantics ", American Anthropologist, 72 (1970), p. 1349-1356. 
voulaient « comprendre la magie avant d'en expliquer l'histoire 9 ». Toutefois, l'exploration de l'histoire de ce concept est menacée par une illusion nominaliste dont les spécialistes sont souvent victimes : l'existence des termes mageía en grec et magia en latin, dont dérive le mot magie, conduit en effet à assimiler ces notions anciennes au concept moderne. Et à éluder la question de la signification qu'elles pouvaient véhiculer pour les Grecs et les Romains, une signification qui peut différer de la conception moderne, fruit d'une longue histoire de sédimentations et d'effacements, et qui peut aussi avoir varié au cours de l'Antiquité. Une recherche sur les représentations grecques et latines associées à la magie semble donc aujourd'hui nécessaire pour retracer l'histoire de cette notion ${ }^{10}$ afin non seulement d'éclairer les cultures antiques mais aussi de faire de ce concept un outil plus pertinent au service des sciences sociales.

\begin{tabular}{l} 
MARCELLO CARASTRO \\
\hline EHESS \\
Centre Louis Gernet \\
2, rue Vivienne \\
F-75002 Paris \\
marcello.carastro@ehess.fr
\end{tabular}

9 Ce propos est tiré des pages préliminaires à l'Esquisse qui apparaissent en appendice dans M. MAUSS, op. cit. : 140 .

10 Une exploration des représentations grecques associées à la notion de mageía est proposée dans M. Carastro, La cité des mages. Penser la magie en Grèce ancienne, Grenoble, éd. J. Millon, coll. Horos, 2006. 